\title{
Brain-Computer Interface Driven Functional Electrical Stimulation System for Overground Walking in Spinal Cord Injury Participant
}

\author{
Christine E. King ${ }^{1}$, Po T. Wang ${ }^{1}$, Colin M. McCrimmon ${ }^{1}$, Cathy C.Y. Chou ${ }^{2}$, An H. Do ${ }^{3}$, \\ and Zoran Nenadic ${ }^{1,4}$
}

\begin{abstract}
The current treatment for ambulation after spinal cord injury (SCI) is to substitute the lost behavior with a wheelchair; however, this can result in many co-morbidities. Thus, novel solutions for the restoration of walking, such as brain-computer interfaces (BCI) and functional electrical stimulation (FES) devices, have been sought. This study reports on the first electroencephalogram (EEG) based BCI-FES system for overground walking, and its performance assessment in an individual with paraplegia due to SCI. The results revealed that the participant was able to purposefully operate the system continuously in real time. If tested in a larger population of SCI individuals, this system may pave the way for the restoration of overground walking after SCI.
\end{abstract}

\section{INTRODUCTION}

The current treatment for ambulation after spinal cord injury (SCI) is to substitute the lost motor behavior with a wheelchair [1]. However, prolonged use of wheelchairs leads to decreased physical activity, which can result in a wide range of co-morbidities, such as heart disease, osteoporosis, and pressure ulcers [2]. Thus, novel biomedical solutions to restore ambulation in these individuals have been sought. Brain-computer interface (BCI) systems may offer one such novel approach by allowing direct brain control of walking via an external device, such as a robotic exoskeleton [3] or a functional electrical stimulation (FES) system [4], [5].

Recent surveys by [6] and [7] suggest that SCI individuals with paraplegia highly prioritize walking as a function that would improve their quality of life if restored. In addition, the survey results in [6] indicated that these individuals would be willing to have a surgery to use an implantable BCI to restore this lost function. To test the feasibility of such a device, an alternative solution is to develop a noninvasive electroencephalogram (EEG) based BCI system for overground walking, as such a system would be a prerequisite for an implantable BCI. To this end, our laboratory integrated an EEG-based BCI with a noninvasive FES system (Parastep I System, Sigmedics, Fairborn, $\mathrm{OH}$ ) for overground walking after paraplegia due to SCI [8]. The following sections will describe this system, and how its performance was

*This work was supported by the National Science Foundation, Award No. 1160200.

${ }^{1}$ C.E. King, P.T. Wang, C.M. McCrimmon, and Z. Nenadic are with the Department of Biomedical Engineering, University of California, Irvine (UCI), Irvine, CA 92697 USA. kingce@uci . edu

${ }^{2}$ C.C.Y. Chou is with the Department of Physical Medicine and Rehabilitation, UCI, Orange, CA 92868 USA

${ }^{3}$ A.H. Do is with the Department of Neurology, UCI, Irvine, CA 92697 USA.

${ }^{4} \mathrm{Z}$. Nenadic is with the Department of Electrical Engineering and Computer Science, UCI, Irvine, CA 92697 USA. assessed over multiple sessions and days by a participant with paraplegia due to SCI.

\section{Methods}

\section{A. Participant Recruitment}

Ethical approval was obtained from the University of California, Irvine Institutional Review Board. The participant was recruited from a population of individuals with complete motor paraplegia due to SCI. The inclusion criteria were those with T6-T11 SCI that were at least 12 months postinjury. Exclusion criteria were severe spasticity, osteoporosis, fractures, unable to tolerate FES, presence of an electronic implant (e.g. pacemaker), presence of pressure ulcers, orthostatic hypotension, poor truncal control, spasms, contractures or orthopedic malformations that may prevent proper use of the FES device, any neuromuscular disease, or cauda equina syndrome. These criteria were assessed through interviews, physical exams, a lower extremity dual energy X-ray absorptiometry (DEXA) scan, X-rays, a pregnancy test, and a tilt-table exam.

\section{B. BCI Walking Simulator Training}

Prior to BCI-FES evaluation, the participant underwent an extensive training procedure to be able to operate both the BCI and Parastep system. To this end, the participant performed BCI walking simulator training described in [9] and [10]. During the BCI walking simulator training sessions, the participant first performed a 10 min procedure in which he alternated between $30 \mathrm{~s}$ epochs of attempted walking and idling while his EEG data were recorded. Then, an EEG decoding model was developed and a brief calibration procedure was performed to find the optimal parameters for real-time operation. Once the model and parameters were determined, the participant then performed 1-5 sessions of a goal-oriented task in a virtual reality environment controlled in real time [9], [10]. Specifically, the participant utilized attempted walking and idling to control the linear ambulation of an avatar while making 10 sequential stops at designated points within the virtual reality environment. Finally, this BCI walking simulator procedure was repeated on each experimental day throughout the entire study.

\section{FES Training}

In accordance with the FDA-approved guidelines for the Parastep system, the participant first reconditioned his muscles prior to gait training. This allowed the user to improve the efficiency of his cardiovascular and respiratory systems, 
as well as reduce the level of muscle atrophy in his lower extremities. To this end, the participant performed strength training with a physical therapist and at-home endurance training with the FES system. Once the physical therapist determined that the participant regained sufficient strength and endurance, and demonstrated the ability to stand using the Parastep, the training sessions progressed to learning the movements required for overground gait training.

The overground gait training using the Parastep system first focused on learning the specific coordination of movements that result in standing, sitting, and walking. Once the participant was able to comfortably perform all of the above tasks, the training progressed until the participant could use manual control switches on the walker to walk the length of the overground walking course $(3.7 \mathrm{~m})$ without fatiguing or any intervention from the physical therapist. Note that this procedure was performed while the participant was mounted in a body-weight support system (ZeroG, Aretech LLC., Ashburn, VA) to prevent any falls.

\section{BCI-Parastep Integration and Movement Sensor Devel- opment}

To facilitate BCI control of the Parastep system, EEG data were wirelessly sent to the computer via a NeXus-32 bioamplifier (Mind Media, Roermond-Herten, The Netherlands), and was subsequently analyzed to determine the individual's intention of either walking or idling (i.e. standing still) in real time. The output decision from the BCI software was then sent to a microcontroller unit (MCU) (Arduino, SmartProjects, Torino, Italy) with wireless communication capabilities (Bluetooth Mate Silver, Sparkfun Electronics, Boulder, CO) and digital relays (Relay shield V2.0, Seeed Technology Inc., Shenzhen, China) that controlled the 3 manual switches on the Parastep system (i.e. sit/stand, left step, and right step). To emulate linear ambulation during intended walking, the step timings from the FES training sessions were analyzed via video recordings, and a custom $\mathrm{C}++$ program was uploaded to the MCU to execute an automatic, cyclic stepping pattern that closely mimicked the FES induced stepping pattern of the participant. Note that this cyclic stepping pattern is intended to mimic how the central pattern generator performs walking in able-bodied individuals. Finally, the MCU only executed these movements when the BCI software switched to the "Walk" state, as determined by the participant's EEG. Conversely, when the BCI software switched to the "Idle" state, the MCU switched the digital relays to perform the standing function of the Parastep system.

In addition to integrating the BCI system with the Parastep device, a movement measurement system was developed to be able to analyze the real-time control of the BCI-Parastep system and to track the position of the participant in real time. To this end, two gyroscopes (L3G4200D, STMicroelectronics, Geneva, Switzerland) and a laser distance meter connected to an MCU (LR3 Laser Rangefinder Interface, Porcupine Electronics LLC., Cedar Park, TX) and mounted on the motor of the ZeroG system were integrated with a master MCU (Arduino). A synchronization signal source was also integrated with the master MCU, and wired to the EEG amplifier of the BCI-Parastep system to be able to align all real-time data for subsequent analysis. Then, via wireless bluetooth communication (Bluetooth Mate Silver), the master MCU sent gyroscope, laser, and synchronization data to the computer in real time. This data was recorded, visualized, and saved using custom written Matlab programs.

\section{E. Suspended Walking Tests}

Once BCI and FES trainings were deemed complete, the participant performed suspended walking tests in which he attempted BCI-Parastep mediated walking and idling while being completely suspended off the ground using the ZeroG system. This was performed to determine whether the participant could use attempted walking and idling to reliably control the BCI-Parastep system without having to maintain balance, shift weight, and perform other difficult movements that are necessary during overground walking. During these tests, the participant was positioned $1 \mathrm{~m}$ from a computer monitor, and the ZeroG unloaded the participant so that his toes were $\sim 5 \mathrm{~cm}$ off the ground. This, along with the Parastep walker, allowed the participant to perform BCI-mediated Parastep walking without moving toward or away from the computer screen. The participant was then instructed by textual cues to perform 5 trials of alternating $30 \mathrm{~s}$ epochs of idling and attempted walking to control the BCI-Parastep system in real time. Finally, the textual cues, $\mathrm{BCI}$ response, and movement data were recorded to assess the performance of this experiment.

\section{F. Overground Walking Course}

The overground walking course consisted of 3 cones positioned $1.8 \mathrm{~m}$ apart (Fig. 1). To test the BCI-Parastep system in a real-time overground walking condition, the participant was instructed to remain idle at each of these cones for 10$15 \mathrm{~s}$, and then to use an attempted walking mental strategy to initiate BCI-Parastep mediated walking to walk to the next cone. Verbal cues were given by the experimenter to help guide the participant through the course, and to help assess the timing of the idling periods. Also, the 10-15 s timing of the verbal cues was randomized by the experimenter to prevent any anticipation by the participant. Note that the ZeroG was used during this task solely to prevent any falls. Finally, the verbal cues (via video recordings), BCI response, and movement data were recorded and saved to assess the performance of the overground walking task.

\section{G. Analysis}

To assess the performance of all tests (i.e. the BCI walking simulator training, suspended walking tests, and overground walking experiments), analysis of the BCI, movement sensors, and cues was performed. Specifically, the performance of the BCI system, as well as the integrated BCI-Parastep system, were analyzed using the aligned data and custom written Matlab programs. Cross-correlation analysis was then performed between the cues and BCI-Parastep mediated response, as determined from the gyroscope data. In addition, 


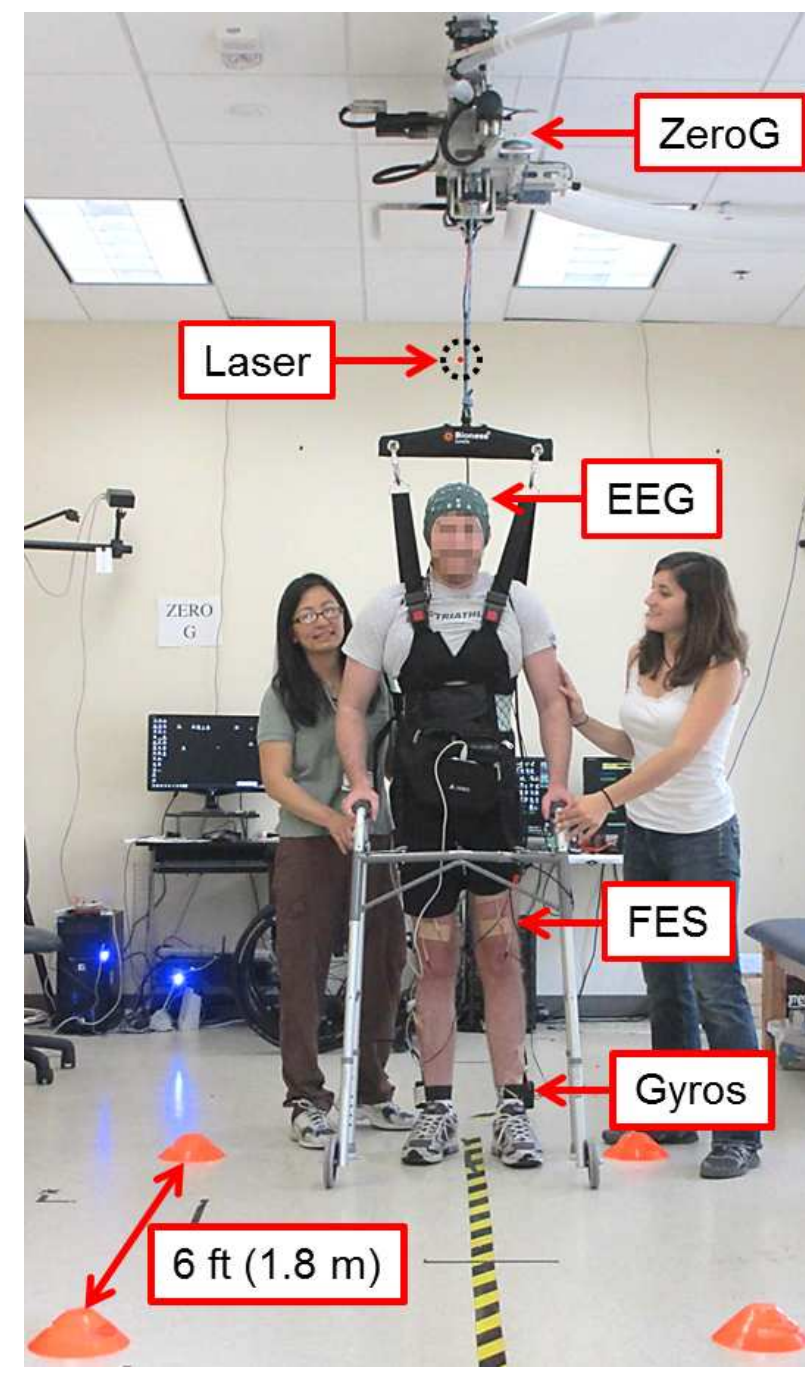

Fig. 1. Partial picture of the overground walking course, depicting the BCI-Parastep system, the movement measurement system (two gyroscopes and a laser distance meter), and the ZeroG. Each cone was positioned $1.8 \mathrm{~m}$ away from each other, and the participant was instructed to idle for 10-15 s, randomized by the experimenter, at each cone.

the number of false alarms and omissions were determined. A false alarm was defined as the initiation of a BCI-Parastep mediated walking response within any intended idling epoch, as determined by the verbal cues. Similarly, an omission was defined as the absence of any BCI-Parastep mediated walking response within any intended walking condition. Finally, Monte Carlo simulations (Section II-G.4) were used to assess whether these performances were purposeful.

1) BCI Walking Simulator Analysis: To assess the performance of the BCI walking simulator training sessions (Section II-B), two performance measures were recorded for each session [9], [10]: (i) the cone score and (ii) the course completion time. A detailed description of these performance measures can be found in [9] and [10]. Briefly, participants were given one point for dwelling at the designated stop for at least $2 \mathrm{~s}$. Only a fraction of a point was given for dwelling at the designated stop between $0.5 \mathrm{~s}$ and 2 $\mathrm{s}$ (increasing proportionally with the dwell time), and no point was given for dwelling less than $0.5 \mathrm{~s}$. A full point was award for dwelling longer than $2 \mathrm{~s}$, but this inherently increased the course completion time. Thus, the maximum cone score was 10 points. Finally, to determine purposeful control, the composite score of the online sessions (ranging from 0 to $100 \%$, where $100 \%$ is a perfect score in both cone score and course completion time) was compared to random walk simulations [10]. This was done by comparing the composite scores to Monte Carlo simulations, and will be further described in Section II-G.4.

2) Suspended Walking Analysis: The suspended walking tests were assessed by performing cross-correlation and information transfer rate (ITR) analyses on the aligned BCI, movement sensors, and video data. Specifically, crosscorrelation analysis between the computer cue and BCIParastep mediated walking and idling was performed to determine the maximal temporal correlation $(\rho)$ and latency (lag). From this data, the number of false alarms and omissions were also calculated. Finally, the ITR between the textual cues and BCI-Parastep mediated response was calculated in bits/s using zero lag [11].

3) Overground Walking Analysis: The overground walking tests were assessed by performing cross-correlation analysis between the verbal cues (as recorded by video data) and the state of the BCI (i.e. "Idle" or "Walk"). In addition to this analysis, cross-correlation analysis between the verbal cues and the gyroscope data was performed. The number of false alarms and omissions were also calculated as explained above. Finally, the control experiments described in the next section were performed to assess purposeful control of each overground BCI-Parastep mediated walking performance.

4) Control Experiments: To assess purposeful control and determine the significance of the online performances, including the BCI walking simulator training sessions, suspended walking tests, and overground walking tests, Monte Carlo simulations were performed. The details of this procedure can be found in [12]. Briefly, the posterior probability of walking given the EEG data was modeled using a nonlinear auto-regressive model with the parameters used during realtime online operation. These simulated probabilities were then fed into the binary state machine of the BCI system, resulting in a simulated sequence of "Idling" and "Walking" states. Cross-correlation was then performed between the intended states, as determined by the walking course or cues given, and the simulated BCI states. This procedure was repeated for a total of 10,000 Monte Carlo runs for each online session. An empirical p-value was then defined as the fraction of Monte Carlo runs that achieved an equal or higher maximum cross-correlation value $(\rho)$ than the participant's.

\section{RESULTS}

One SCI individual met all evaluation criteria (Section IIA) and participated in the study. The participant was an active 26-year-old male with paraplegia (AIS B, $>5$ years post injury). 


\section{A. BCI Walking Simulator}

The participant's BCI walking simulator training results demonstrated that the EEG decoding models' performances improved over time. These EEG decoding models also revealed that the participant mostly utilized features in the low$\beta(13-20 \mathrm{~Hz})$ and high $-\beta(20-30 \mathrm{~Hz})$ frequency bands over the $\mathrm{C} 3$ and $\mathrm{C} 4$ electrodes, and over $\mathrm{Cz}$ and $\mathrm{CPz}$, respectively (Fig. 2).

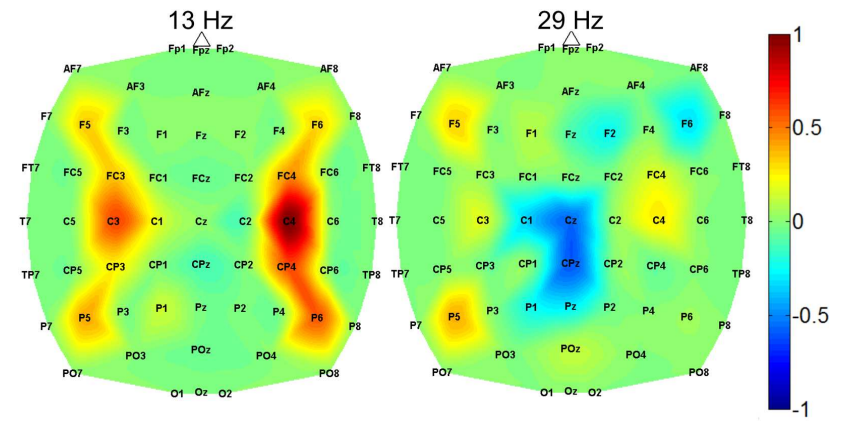

Fig. 2. Typical EEG feature extraction maps on one experimental day, showing salient features (in blue, -1 , and red, +1 ) over the $\mathrm{C} 3$ and $\mathrm{C} 4$ electrodes in the low $-\beta$ band, and the $\mathrm{Cz}$ and $\mathrm{CPz}$ electrodes in the high $-\beta$ band.

The walking simulator goal-oriented task described in Section II-B and in [9], [10] demonstrated that the participant was able to purposefully operate the BCI system in real time on the $1^{\text {st }}$ experiment day. The participant's composite score improved over time, and eventually approached $100 \%$ by the $11^{\text {th }}$ experiment day (Fig. 3). Finally, note that the participant performed this task on each experimental day throughout the entire study to ensure that he could maintain control of the BCI system.

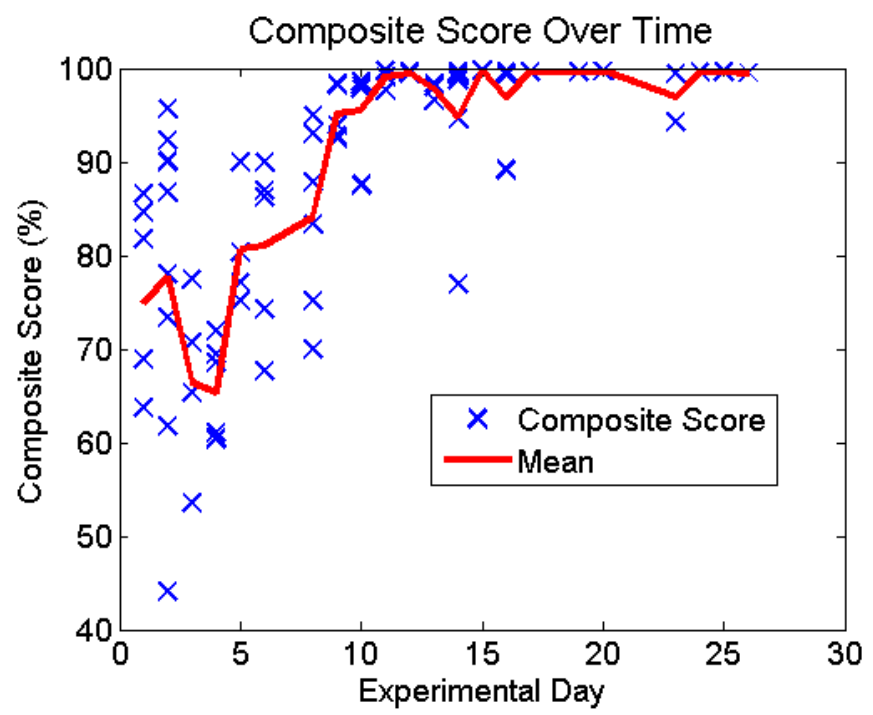

Fig. 3. Composite scores and the mean for the BCI walking simulator training sessions, as described in [9], [10] and Section II-G.1.

\section{B. Suspended Walking Tests}

The results of the suspended walking tests revealed that the participant was able to operate the BCI-Parastep system in real-time with a very high correlation and ITR, averaging 0.937 and $2.127 \mathrm{bits} / \mathrm{s}$, respectively. The average lag between the computer cues and BCI-Parastep mediated walking was $3.63 \mathrm{~s}$. In addition, the participant had no omissions during each suspended walking test, and only had 1 false alarm that lasted $1.75 \mathrm{~s}$ during the $1^{\text {st }}$ suspended walking test. Finally, note that the empirical p-value was $<10^{-4}$ for both suspended walking tests performed (as determined in Section II-G.4).

\section{TABLE I}

PERFORMANCES OF THE SUSPENDED WALKING TESTS.

CROSS-CORRELATION ( $\rho$, LAG), ZERO-LAG ITR, FALSE ALARMS (FA

AND DURATION, DUR.) BETWEEN CUES AND PARTICIPANT'S BCI-PARASTEP MEDIATED WALKING.

\begin{tabular}{|c|c|c|c|c|c|}
\hline $\begin{array}{c}\text { Exp. } \\
\text { Day }\end{array}$ & $\rho$ & $\begin{array}{c}\text { Lag } \\
(\mathrm{s})\end{array}$ & FA & $\begin{array}{c}\text { FA Dur. } \\
(\mathrm{s})\end{array}$ & $\begin{array}{c}\text { ITR } \\
(\mathrm{bits} / \mathrm{s})\end{array}$ \\
\hline 18 & 0.917 & 3.00 & 1 & 1.75 & 2.150 \\
19 & 0.957 & 4.25 & 0 & 0 & 2.103 \\
\hline Avg. & 0.937 & 3.63 & 0.5 & 0.88 & 2.127 \\
\hline
\end{tabular}

\section{Overground Walking Tests}

The participant was able to perform the BCI-Parastep overground walking tests after the suspended walk tests on the $20^{\text {th }}$ experiment day. An example figure of this overground walk test on the $23^{\text {rd }}$ experiment day can be seen in Fig. 4. It can be noted that this session resulted in no omissions, and only one very brief false start prior to the verbal cue during the $2^{\text {nd }}$ walk trial. Furthermore, after comparing the $\mathrm{BCI}$ response and gyroscope data to the verbal cues, the participant was able to perform BCI-Parastep mediated walking with a very short response time between the verbal cue and walking/idling states (see Table II). Finally, the gyroscope data indicated that the BCI state was able to perfectly control the Parastep mediated walking, resulting in advancement down the course to each cone.

The overground walking analyses resulted in purposeful performances and high correlations across all sessions and experimental days (Table II). Specifically, the Monte Carlo simulations resulted in a p-value $<10^{-4}$ for all experiment days and sessions, except for the $24^{\text {th }}$ experiment day ( $\mathrm{p}$ value $=0.0369$ ). The experiment day that resulted in an insignificant $\mathrm{p}$-value was due to the inability of the participant to switch to an idle state using the BCI system, resulting in a low correlation between the verbal cues and BCI-Parastep mediated response. Overall, the participant had no omissions during all overground walk tests, and very few false alarms (Table II).

\section{DISCUSSION}

The results of this study show that BCI driven overground walking in an SCI individual is feasible. Through both the suspended walk tests and overground walking course using the BCI-Parastep system, it was proven that this SCI 


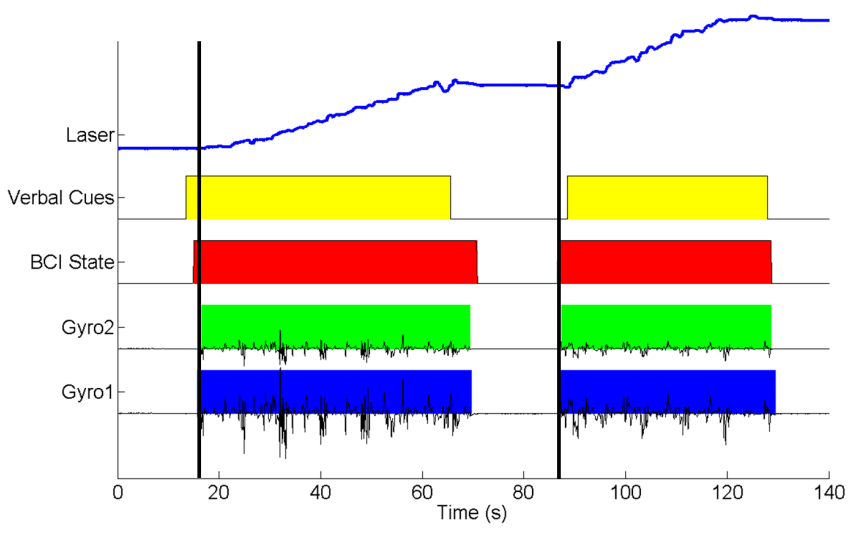

Fig. 4. The overground walking test results for the $23^{\text {rd }}$ experiment day. Depicted here are the laser data, verbal cues given by the experimenter, the state of the BCI system, and the gyroscope data used to assess the BCIParastep mediated walking performance. Note that there is $4 \mathrm{~s}$ between each FES mediated step to allow for weight shifting and walker advancement.

TABLE II

Performances of overground walking tests (aVerage, AVg., STANDARD DEVIATION, S.D.). CROSS-CORRELATION ( $\rho$, LAG), TOTAL NUMBER OF FALSE ALARMS (FA) BETWEEN VERBAL CUES AND BCI-PARASTEP MEDIATED WALKING.

\begin{tabular}{|c|c|c|c|c|}
\hline $\begin{array}{c}\text { Exp. } \\
\text { Day }\end{array}$ & $\begin{array}{c}\text { No. } \\
\text { Sess. }\end{array}$ & $\rho$ & $\begin{array}{c}\text { Lag } \\
(\mathrm{sec})\end{array}$ & $\begin{array}{c}\text { Total } \\
\text { No. FA }\end{array}$ \\
\hline 20 & 1 & 0.429 & 6.547 & 6 \\
21 & 1 & 0.674 & 6.113 & 1 \\
22 & 2 & $0.762,0.554$ & $1.992,2.344$ & 7,4 \\
23 & 1 & 0.909 & 1.555 & 1 \\
24 & 2 & $0.397,0.369$ & 0,15 & 2,2 \\
25 & 1 & 0.520 & 11.434 & 4 \\
26 & 4 & $0.792 \pm .022$ & $1.787 \pm 0.791$ & $4 \pm 2.160$ \\
\hline Avg. & 1.714 & 0.649 & 4.344 & 3.583 \\
S.D. & 1.113 & 0.186 & 4.632 & 2.151 \\
\hline
\end{tabular}

individual was able to produce and maintain a high level of control of the system during both walking and idling states across experimental days. Specifically, the suspended walk tests resulted in high ITRs and correlations, as well as very low false alarm and omission rates. This translated into purposeful real-time control of the BCI-Parastep system during the overground walking tests.

Although the participant was able to achieve a high level of control during both the suspended and overground walking tests, the number of false alarms increased and correlation values decreased when progressing from the suspended to the overground walking tests. This may be due to issues with postural stability and the participant's need to use his arms to maintain balance during weight loading. Since the EEG decoding model shown in Fig. 2 revealed salient features over the arm representation areas of the brain, this region may have been similarly activated when the participant used his arms during weight loading for postural stability. Furthermore, the insignificant p-value on the $24^{\text {th }}$ experiment day was likely due to the fact that the optimal parameters for both the BCI and Parastep systems were not yet determined. Note that the conditions of weight loading during the overground walk tests are much different than the suspended walk tests and BCI walking simulator training, which is why optimizing these parameters was very difficult. Furthermore, it was difficult to obtain multiple sessions of the overground walking tests because of the high level of fatigue from the Parastep system, and the need for the FES to compensate for weight loading. However, the participant's ability to perform multiple sessions improved over time, approaching 4 purposeful sessions with a high level of control on the final experiment day.

\section{CONCLUSION}

This study demonstrates for the first time that a BCI driven FES system for overground walking can be operated by a paraplegic individual with SCI. The participant was able to purposefully operate the device in real time using an attempted walking and idling strategy after only minimal training, and was able to maintain this level of control throughout the study. If tested in a larger SCI population, this system may demonstrate that BCI driven overground walking is feasible. However, future studies may require invasive recording techniques to ensure robust and longterm operation of the system, which may result in a novel neuroprosthesis for the restoration of walking in those with complete motor SCI. Finally, for those with incomplete motor SCI, the system presented in this study may become a novel noninvasive neurorehabilitative therapy for overground walking, and may potentially restore this lost function to these individuals.

\section{REFERENCES}

[1] B.H. Dobkin. The Clinical Science of Neurologic Rehabilitation. Oxford University Press, $2^{\text {nd }}$ edition, 2003.

[2] R.L. Johnson, C.A. Brooks, and G.G. Whiteneck. Cost of traumatic spinal cord injury in a population-based registry. Spinal Cord, 34(8):470-480, 1996.

[3] P. Jaspers, L. Peeraer, W. Van Petegem, and G. Van der Perre. The use of an advanced reciprocating gait orthosis by paraplegic and individuals: a follow-up study. Spinal Cord, 35:585-589, 1997.

[4] D. Graupe, R. Davies, H. Kodylewski, and K.H. Kohn. Ambulation by traumatic T4-12 paraplegics using functional neuromuscular stimulation. Critical Review Neurosurgery, 8:221-231, 1998.

[5] D. Graupe and K.H. Kohn. Functional neuromuscular stimulator for short-distance ambulation by certain thoracic-level spinal-cord-injured paraplegics. Surgical Neurology, 50(3):202-207, 1998.

[6] J.L. Collinger, M.L. Boninger, T.M. Bruns, K. Curley, W. Wang, and D.J. Weber. Functional priorities, assistive technology, and brain-computer interfaces after spinal cord injury. The Journal of Rehabilitation Research and Development, 50(2):145-159, 2013.

[7] K.D. Anderson. Targeting recovery: Priorities of the spinal cordinjured population. Journal of Neurotrauma, 21(10):1371-1383, 2004.

[8] C.E. King, C.M. McCrimmon, P.T. Wang, C.C.Y. Chou, Z. Nenadic, and A.H. Do. Brain-computer interface driven functional electrical stimulation system for overground walking: A case report. In American Academy of Neurology Annual Meeting, 2014.

[9] P.T. Wang, C.E. King, L.A. Chui, A.H. Do, and Z. Nenadic. Self-paced brain-computer interface control of ambulation in a virtual reality environment. Journal of Neural Engineering, 9(5):056016, 2012.

[10] C.E. King, P.T. Wang, L.A. Chui, A.H. Do, and Z. Nenadic. Operation of a brain-computer interface walking simulator for individuals with spinal cord injury. Journal of NeuroEngineering and Rehabilitation, 10(77):1-14, 2013.

[11] T. Cover and J. Thomas. Elements of Information Theory. Wiley Interscience, 1991.

[12] A.H. Do, P. T. Wang, C. E. King, S.N. Chun, and Z. Nenadic. Brain-computer interface controlled robotic gait orthosis. Journal of NeuroEngineering and Rehabilitation, 10(111), 2013. 